\title{
A Methodology to Generate a Time-varying Adjustable Wave Impedance inside a TEM Cell
}

\author{
Guanghua Li, V. A. K. Prabhala, Abhinav Saxena, Qian Wang, Pratik Maheshwari, David Pommerenke \\ Department of Electrical and Computer Engineering \\ Missouri University of Science and Technology \\ Rolla, MO, USA. \\ glbm4, vkpzvf, arsnt7, qwk7d, prm8c7, davidjp@mst.edu
}

\begin{abstract}
A methodology to generate a time-varying adjustable wave impedances inside a TEM cell is introduced for electromagnetic susceptibility tests of small electronic modules. The TEM cell is excited by two amplified arbitrary waveforms. By varying the excitation waveforms and their timing, the momentary wave impedance and field strength inside the TEM cell can be adjusted.
\end{abstract}

Keywords-Arbitrary waveform; broadband amplifiers; susceptibility test; wave impedance; TEM cell

\section{INTRODUCTION}

Electronic modules in automobiles may be exposed to radio frequency noises. The field strengths from the noise sources are 100's of $\mathrm{V} / \mathrm{m}$ [1]. The wave impedance of the fields is unknown, because either electric field or magnetic field can be dominant near the module surfaces. If a module is only tested in a matched transverse electromagnetic (TEM) cell, then it is tested for $377 \Omega$ wave impedance which may not reflect the fields in real applications. The module also can be tested in a mode stirred chamber with strongly varying field impedances. However, the wave impedance is not known easily at the location of the module.

The authors of [2] varied the load impedance of a TEM cell to obtain various known field strengths and wave impedances inside a TEM cell. The authors of [3] [4] excited the TEM cell at both ports using sinusoidal waveforms.

This paper introduces a technique where arbitrary timedomain signals drive the TEM cell at both ends. This generates desired time domain waveforms with the desired field strength, and thus results in arbitrary wave impedance within the TEM cell. This technique allows engineers not only to investigate the robustness of a module, but also to understand if the coupling mechanism is electric or magnetic coupling. Section II describes the principle of generating dominant $\mathrm{E}$ - and $\mathrm{H}$-fields. Section III introduces a wideband two-channel power amplifier, with maximal output power of 10.7 Watts from $2 \mathrm{MHz}$ to $4 \mathrm{GHz}$. Section IV describes the measurement procedure to validate the arbitrary wave impedance within the TEM cell.

\section{GEENRATION OF DOMINANT E AND H FIELDS}

The basic method for generating fields of adjustable wave impedance is shown in Fig. 1. For the analysis the TEM cell is considered as a lossless two-wire transmission line of length $l$ with characteristic impedance $Z_{0}$. The wave velocity inside the TEM cell is given by $\mathrm{v}_{0}$. The TEM cell is driven simultaneously by two arbitrary signals $f(t)$ and $g(t)$. At an observation point inside the TEM cell, $P(x=0, y=d / 2, z=l / 2)$, the total voltage of the septum against the ground is given by,

$$
V\left(z=\frac{l}{2}, t\right)=\frac{1}{2} f\left(t-\frac{l}{2 v_{0}}\right)+\frac{1}{2} g\left(t-\frac{l}{2 v_{0}}\right)
$$

Here it is assumed that the TEM cell is matched at both source and load ends; that is, $\mathrm{ZS}=\mathrm{ZL}=\mathrm{Z} 0$. From the voltage in (1), the time-varying electric field between the septum and the ground is given by,

$$
E\left(x=0,0<y<d, z=\frac{l}{2}, t\right)=\frac{1}{2 d}\left\lfloor f\left(t-\frac{l}{2 v_{0}}\right)+g\left(t-\frac{l}{2 v_{0}}\right)\right\rfloor \hat{a}_{y}
$$

The magnetic field is calculated from the electric field as,

$$
H\left(x=0,0<y<d, z=\frac{l}{2}, t\right)=-\frac{1}{2 d \eta_{0}}\left\lfloor f\left(t-\frac{l}{2 v_{0}}\right)-g\left(t-\frac{l}{2 v_{0}}\right)\right\rfloor \hat{a}_{x}
$$

where $\eta_{0}=377 \Omega$ is the intrinsic wave impedance of free space. Once the electric and magnetic fields are obtained, the wave impedance in the TEM cell is given by

$$
\begin{aligned}
& \eta\left(x=0,0<y<d, z=\frac{l}{2}, t\right) \\
& =\left|E\left(x=0,0<y<d, z=\frac{l}{2}, t\right) / H\left(x=0,0<y<d, z=\frac{l}{2}, t\right)\right| \\
& =\eta_{0}\left|f\left(t-\frac{l}{2 v_{0}}\right)+g\left(t-\frac{l}{2 v_{0}}\right)\right| /\left|f\left(t-\frac{l}{2 v_{0}}\right)-g\left(t-\frac{l}{2 v_{0}}\right)\right|
\end{aligned}
$$


A TEM cell model was selected to validate the wave impedance in (4). The TEM cell's cross-section is shown in Fig. 2 and the two ports are shown in Fig. 1. The measured reflection is less than $10 \mathrm{~dB}$ up to at least $4 \mathrm{GHz}$, while the measured transmission is smooth without significant resonance in the same frequency range in Fig. 3. To excite the TEM cell in a CST simulation [5], a Gaussian signal, $\mathrm{s}(\mathrm{t})$, was used, as depicted in Fig. 4. The peak voltage of the Gaussian signal was $14.14 \mathrm{~V}$. Multiple simulations were performed to generate different dominant fields and wave impedances in the TEM cell.

In the first case, the two TEM cell ports, as shown in Fig. 1, were fed by signals $f(t)$ and $g(t)$, which were given as $f(t)=s(t)$ and $g(t)=0$, respectively. This emulates the general application of a TEM cell, where only one travelling wave with a wave impedance of $377 \Omega$ is created. The simulated fields were monitored using probes in the CST at the observation point $P$ $(x=0, y=d / 2, z=l / 2)$ as shown in Fig. 1 . The wave impedance is $378 \Omega$ at $t=0.3 \mathrm{~ns}$, calculated from the simulated E- and $\mathrm{H}-$ fields as shown in Fig. 5.

In the second case, the signals are $f(t)=g(t)=s(t)$ to generate dominant electric fields with wave impedances of infinity. The calculated peak electric field, from (2), is $1.35 \mathrm{kV} / \mathrm{m}$ at the observation point, while the simulated peak is $1.27 \mathrm{kV} / \mathrm{m}$ in Fig. 5a. The difference between the calculated and simulated peak field strength is caused by the mismatch between the TEM cell characteristic impedance and the port impedance in the simulation. The simulated magnetic field is zero. Therefore, when the two excitations are of the same magnitude and in phase, the electric field is dominant over the magnetic field. This can also be explained in terms of mode analysis. When the TEM cell is evenly excited, the plane perpendicular to the septum and across the observation point has an open boundary condition. The total current is then zero. The magnetic field around the septum is zero, while the electric field is maximal. Therefore, the electric field is dominant over the magnetic field.

In the third case, the two excitations are of the same magnitude but out of phase; that is, $f(t)=-g(t)=s(t)$. At the observation point, the electric field is zero, while the magnetic field is maximal, as shown in Fig. 5. The wave impedance in (4) is zero. The magnetic field is dominant over the electric field. Ideally, the achievable field wave impedance varies from zero to infinity in the TEM cell. In reality, the achievable wave impedance will depend upon how close are the magnitudes and phases of in-phase (out-of-phase) excitations, $f(t)$ and $g(t)$.

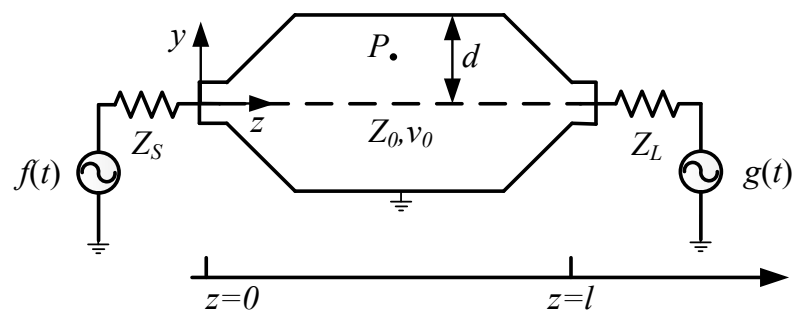

Fig. 1. Side view of the TEM cell with two simultaneous excitations to generate dominant $\mathrm{E}$ - and $\mathrm{H}$-fields.

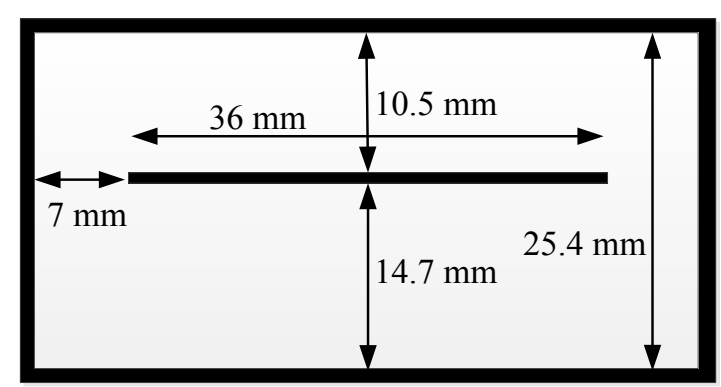

Fig. 2. Cross-section view of a simple TEM cell model in CST.

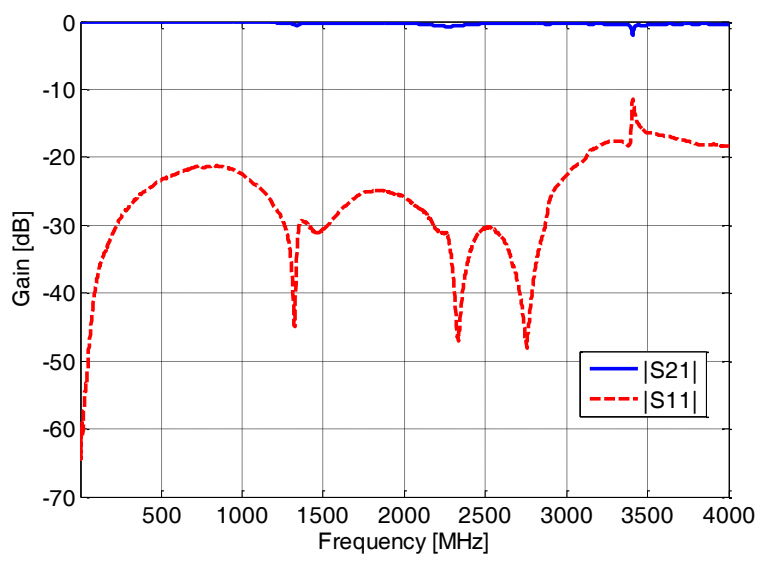

Fig. 3. Measured reflection and transmission coefficients of the TEM cell with geometry dimensions in Fig. 2.

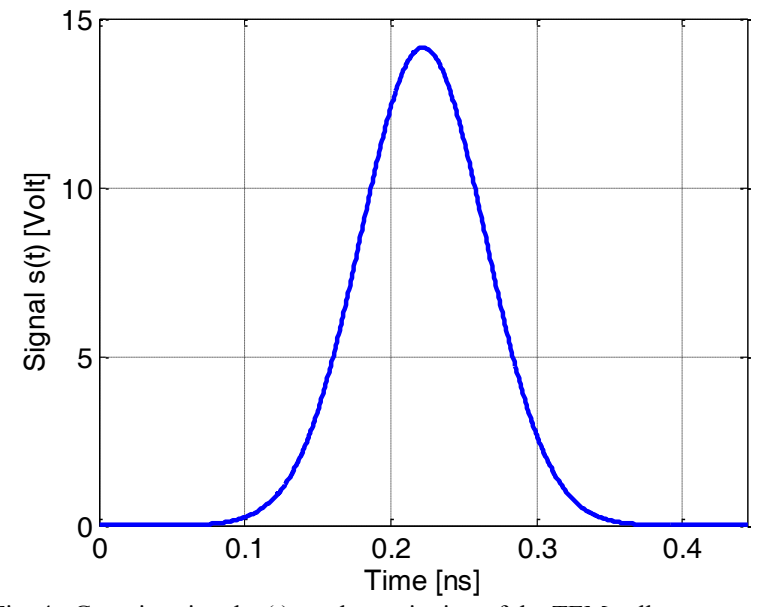

Fig. 4. Gaussian signal, $s(t)$, as the excitation of the TEM cell.

It can be inferred that the excitations, $f(t)$ and $g(t)$, are not limited to time-harmonic signals in the steady state; they can be any time-varying signals. Thus, any arbitrary fields and wave impedances can be generated in the TEM cell, which is useful in EMC tests to emulate various near-field environments in the time domain. The limit is the bandwidth and power of the amplifier used. For achieving fast rise times (100ps) the bandwidth needs to be $>3 \mathrm{GHz}$, but for achieving long pulses the lower cut off frequency needs to be as low as possible. 


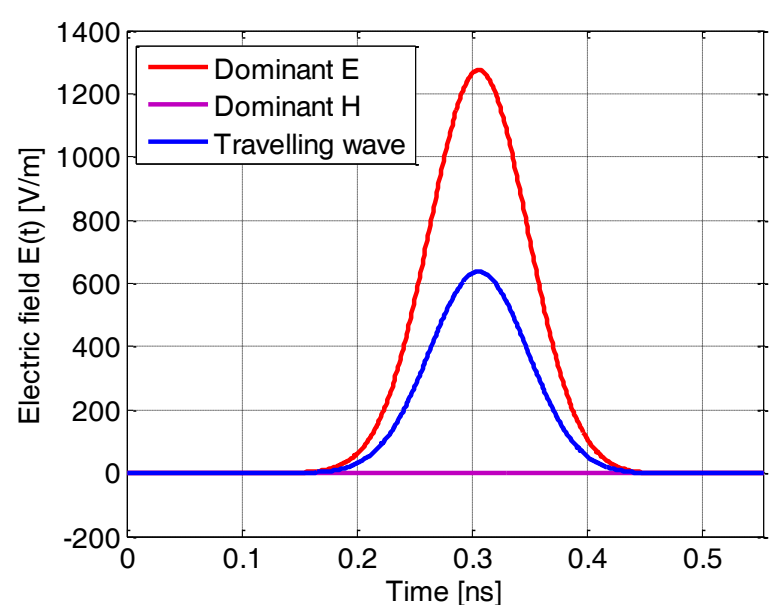

(a)

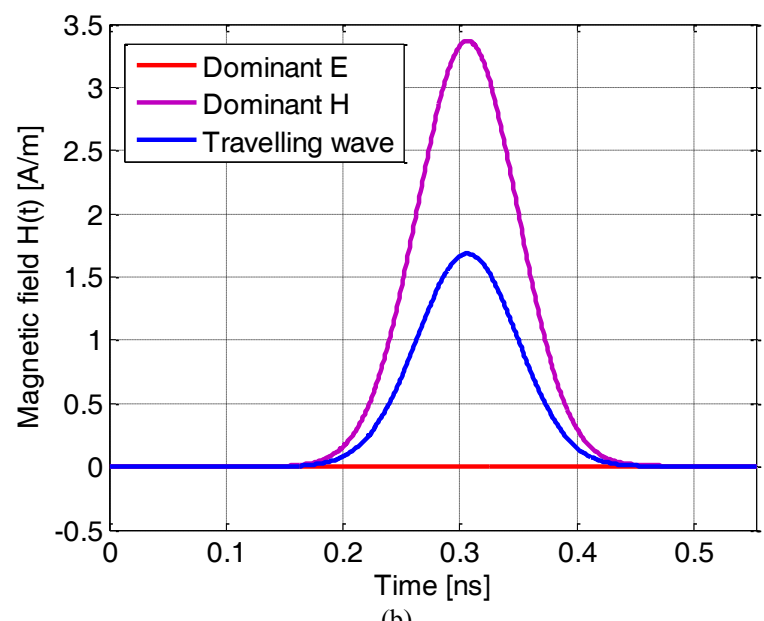

(b)

Fig. 5. Time-varying electric and magnetic field at the observation point, (a) electric fields and (b) magnetic fields.

The next section describes details of an amplifier developed for driving the TEM cell.

\section{POWER AMPLIFIER TO STRENGTHEN FIELD STRENGTH}

In order to generate strong field strengths in the TEM cell, a two-channel power amplifier system was designed to operate from $2 \mathrm{MHz}$ to $4 \mathrm{GHz}$, with a maximal output power of $10.7 \mathrm{~W}$ per channel. A $3 \mathrm{~dB}$ attenuator was placed at the channel output, or else the full power level of one amplifier would be fed into the other channel. This would damage the $\mathrm{GaN}$ transistor. Each one of the two channels includes four stages of amplifiers, as shown in Fig. 6a. The first three stages of amplifiers, with part numbers GVA-62+ [6] and AM003536SF-2H-S [7], are preamplifiers to provide enough driving power to the fourth amplifier, CREE GaN with part number CMPA0060025F C13453S [8], which is a GaN HEMT based MMIC power amplifier.

The gain of the amplifiers is not flat but rolls-off due to parasitics and the transistors used at high frequencies. To reduce the gain roll-off of $8.5 \mathrm{~dB}$ from $2 \mathrm{MHz}$ to $4 \mathrm{GHz}$, a gain slope equalizer was designed based on a PI-type attenuator. Although a single equalizer can achieve a gain variation of $8.5 \mathrm{~dB}$, a series of $4 \mathrm{~dB}$ equalizers were selected. This provides more freedom of placing them between different stages to not only compensate gain, but to avoid saturation of any driver stage.

A logic control board was designed to protect the GaN, which requires a correct sequencing during turn on and off, in Fig. 6c. The gate voltage is supplied to the GaN before the drain voltage is turned on/off and is continuously monitored. Once the gate voltage exceeds (= not negative enough) the preset threshold value, the drain voltage supply is turned off. Also, when the drain current exceeds the maximal allowable value, the drain voltage supply is shut down.

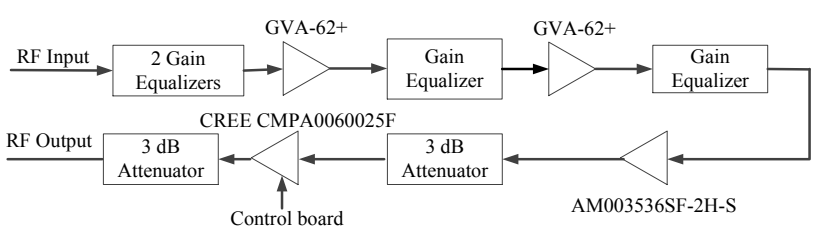

(a)

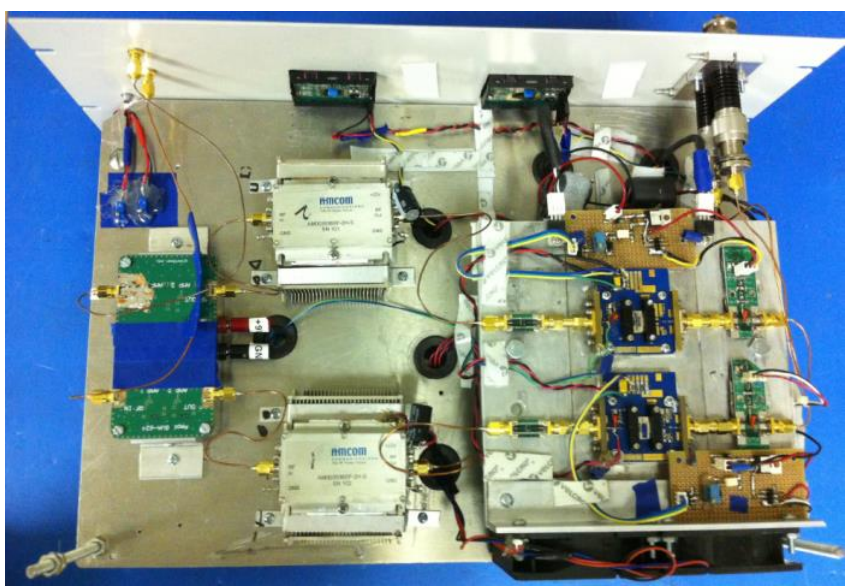

(b)

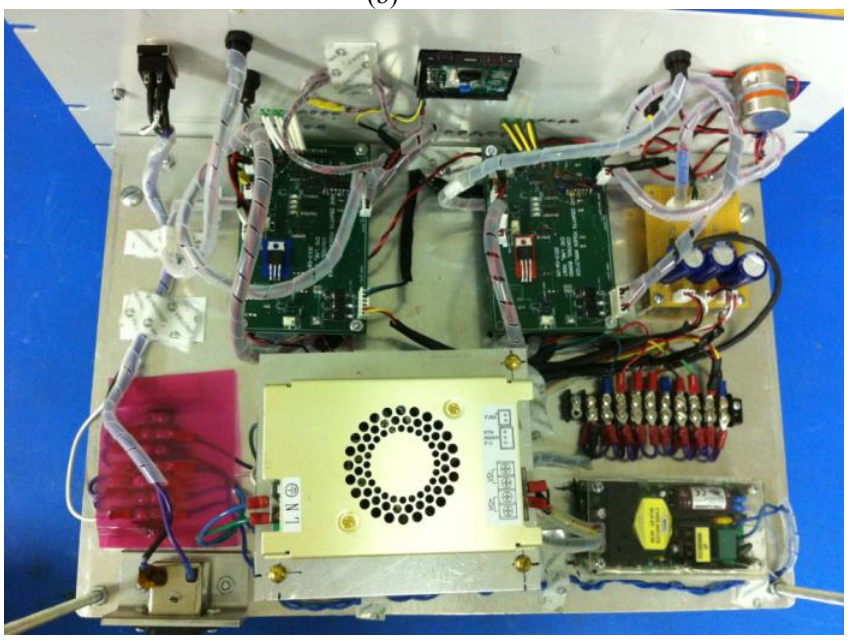

(c)

Fig. 6. Two-channel power amplifier system, (a) block diagram, (b) power amplifiers, and (c) logic control boards and power supplies. 
The measured gain for both channels of the power amplifier is shown in Fig. 7. The median gain is $52 \mathrm{~dB}$ from $2 \mathrm{MHz}$ to $4 \mathrm{GHz}$. The saturated power is $40.3 \mathrm{dBm}(10.7 \mathrm{~W})$ and $40 \mathrm{dBm}(10 \mathrm{~W})$, at $10 \mathrm{MHz}$ and $100 \mathrm{MHz}$, respectively, when the drain voltage $\mathrm{V}_{\mathrm{DD}}$ is $46.7 \mathrm{~V}$ and the quiescent current $\mathrm{I}_{\mathrm{DQ}}$ is $500 \mathrm{~mA}$. At higher frequencies, the drain current reached the pre-set 1 A limit, causing the power amplifier shutting down. The saturated power is not available. When the input power to one port of the TEM cell is $10.7 \mathrm{~W}$ and the other port of the TEM cell is terminated to a matched load $\left(Z_{S}=Z_{L}=Z_{0}=50\right.$ $\Omega$ ), the peak electric field at the observation point can be calculated as $3.12 \mathrm{kV} / \mathrm{m}$. When both ports of the TEM cell are driven with in-phase signals of $12.5 \mathrm{~W}$, the peak electric field can be calculated as $6.24 \mathrm{kV} / \mathrm{m}$ using (2).

\section{EXPERIEMENTAL APPROACH AND INSTRUMENTATION}

Two pulses from a programmable arbitrary waveform generator drive the two ports of the TEM cell simultaneously after amplification. The magnitude and phase of the pulses can be adjusted for setting the dominant field impedance in the TEM cell. The input pulse to the amplifier which is used for obtaining a high wave impedance is shown in Fig. 8a. The pulse is first amplified by the amplifier and then attenuated by a $20 \mathrm{~dB}$ attenuator, which protects the oscilloscope measuring the amplified pulse. In order to obtain the same amplitude and phase of the output waveform from the second channel, the magnitude of the other excitation pulse into the second channel is tuned due to different gains that are shown in Fig. 7. Fig. 8a shows that both channels have similar waveforms, which will be injected into the TEM cell at the same time. Similarly, two pulses out of phase are generated for low wave impedance and shown in Fig. 8 b.

Fig. 9 shows the measurement setup used to validate the dominating field in the TEM cell. A $1.5 \mathrm{~cm}$ long microstrip trace on a PCB having a characteristic impedance of $50 \mathrm{Ohms}$ completes the TEM cell. The microstrip trace is inside the TEM cell. The trace has two SMA connectors at its ends for monitoring the induced voltages by an oscilloscope.

Fig. 10 shows the in-phase voltages measured at the microstrip trace ends. When the electric field is dominant, the

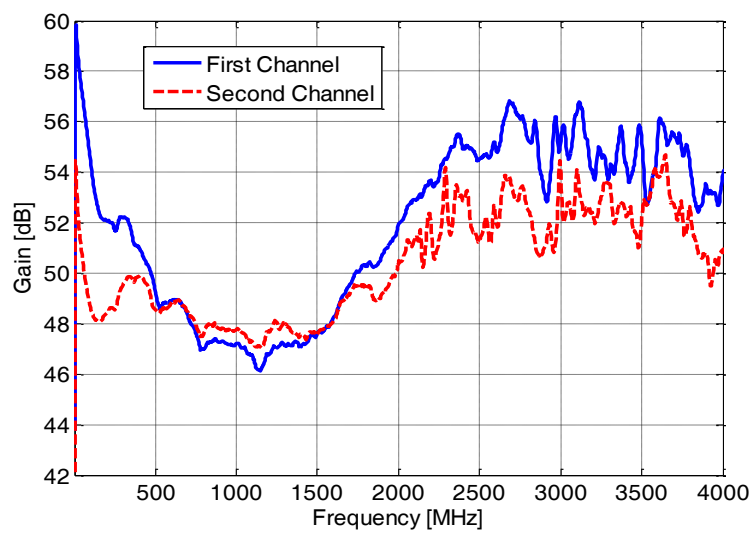

Fig. 7. Gain of the power amplifier (block diagram in Fig. 6a.)

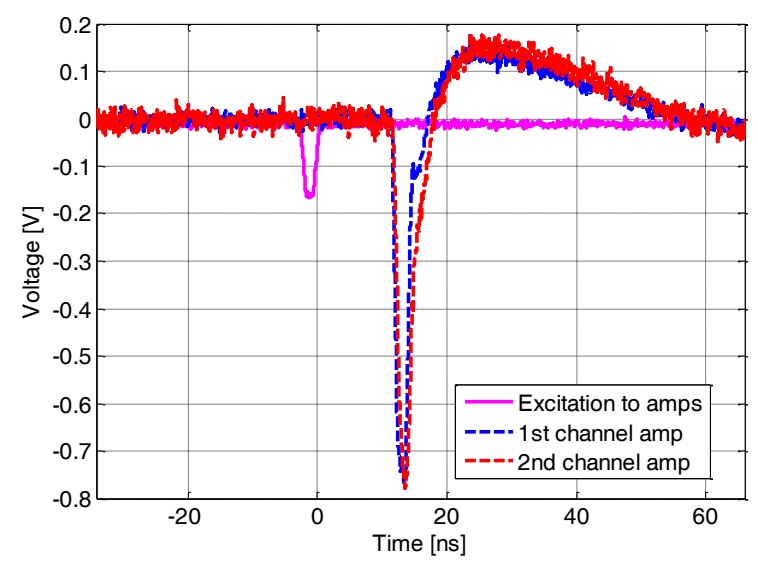

(a)

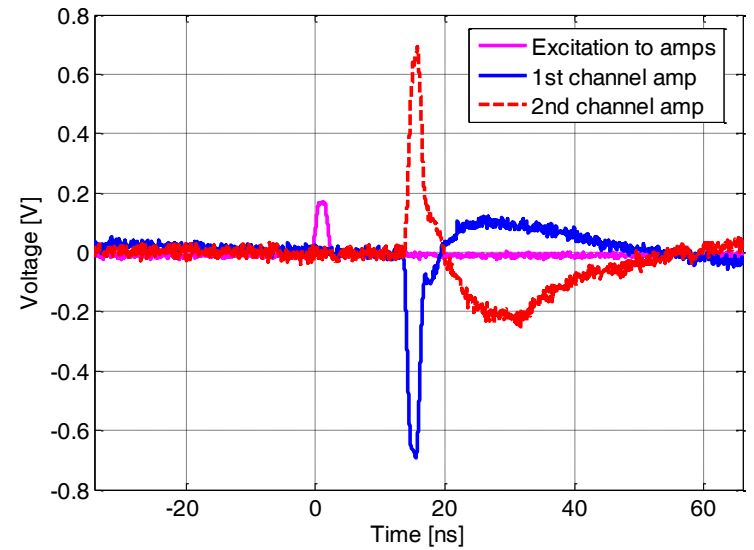

(b)

Fig. 8. Waveform from arbitrary wave generator (pink) and amplified waveforms to be injected into the TEM cell (red and blue), (a) amplified pulses from two channels are in phase to generate dominant electric fields, and (b) amplified pulses from two channels are out of phase to generate dominant magnetic fields.

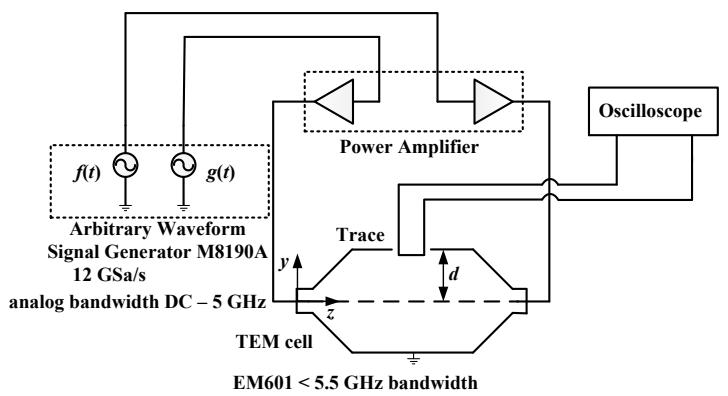

Fig. 9. a) Block diagram of instrumentation for generating time-varying

coupling from the TEM cell septum to the microstrip trace is mainly capacitive. The same voltage and polarity is measurable at both ends of the microstrip. The time derivative of the field causes the coupling. The pulses at $10 \mathrm{~ns}$ and $15 \mathrm{~ns}$ are caused by the rising and the falling edge of the excitation pulses, Fig. 10. When the magnetic field is dominant, the induced voltages at the microstrip trace will be out of phase, as shown in Fig. 11. The data shows that high and low impedances are generated inside the TEM cell. 


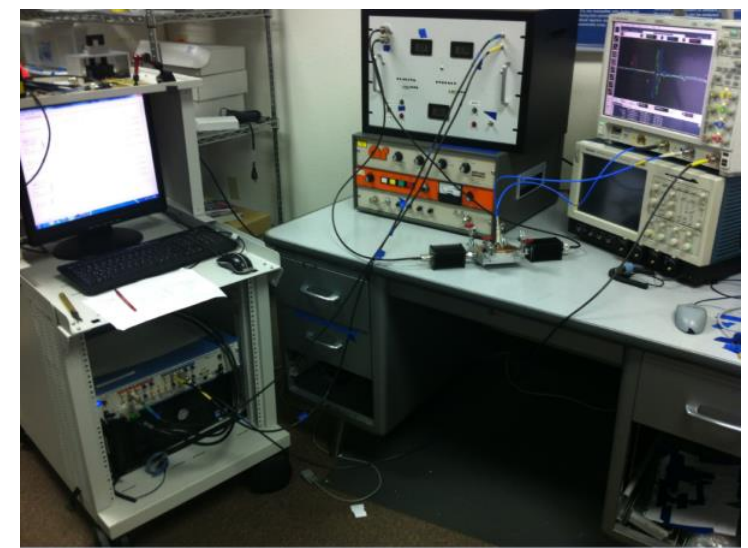

Fig. 9. b) Picture showing the arbitrary waveform signal generator (Agilent M8190A) having $12 \mathrm{GSa} / \mathrm{sec}$ and an analog bandwidth of DC $5 \mathrm{GHz}$.

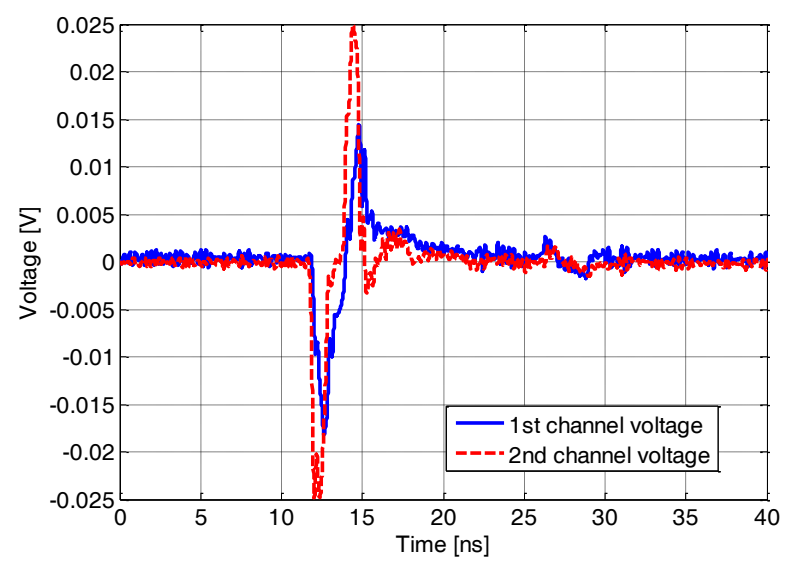

Fig. 10. Induced in-phase voltages at the microstrip trace for high wave impedance.

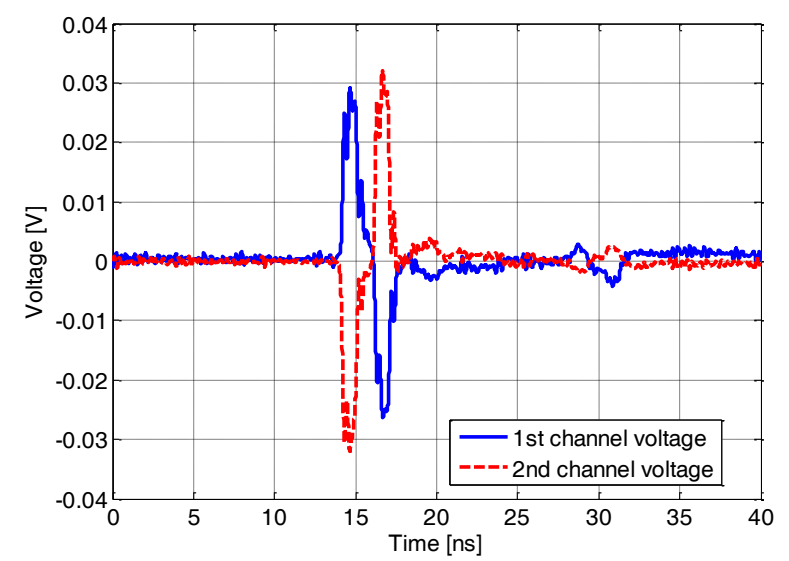

Fig. 11. Induced out-of-phase voltages at the microstrip trace for low wave impedance.

\section{CONCLUSION}

A methodology is proposed to generate time-varying fields and wave impedances inside a TEM cell, which is excited by two arbitrary signals simultaneously. The operator can select the waveform, field strengths and wave impedances within the limitation of the two channel amplifier to test the susceptibility of small modules against the fields the module may experience at its final location. Further, a power amplifier design offering maximal $10.7 \mathrm{~W}$ from $2 \mathrm{MHz}$ to $4 \mathrm{GHz}$ is described in the paper.

\section{ACKNOWLEDGMENT}

This material is based upon work supported by the National Science Foundation under Grant No. 0855878. We want to thank CREE for providing two GaN MMIC modules and the time spent to support us.

\section{REFERENCES}

[1] M. Steffka, "Automotive EMC Introduction and Overview," [Online]. Available: http://www.cvel.clemson.edu.

[2] M. T. Ma , E. B. Larsen, and M. L. Crawford, "Electromagnetic fields with arbitrary wave impedances generated inside a TEM cell," IEEE Trans. Electromagn. Compat., vol. 33, no. 4, pp. 358-362, Nov. 1991.

[3] S. K. Das , V. Venkatsan , B. K. Sinha, and G. Uma, "A technique of electromagnetic interference measurements with high-impedance electric and low-impedance magnetic fields inside a TEM cell," in IEEE Int. Symp. Electromagn. Compat. Washington, DC, 1990, pp. 367-369.

[4] S. Jeon, S. Park, and D. Kim, "Simple method to generate dominant Eand H-fields inside a four-port TEM cell," IEEE Trans. Electromagn. Compat., vol. 55, no. 5, pp. 979-982, Oct. 2013.

[5] CST Microwave studio. [Online]. Available: http://www.cst.com

[6] MiniCircuits. [Online]. Available: http://www.minicircuits.com.

[7] AMCOM. [Online]. Available: http://www.amcomusa.com

[8] CREE. [Online]. Available: http://www.cree.com 